\title{
A NEW APPROACH TO MODELLING IN PLANNING OF ELECTRIC POWER SYSTENS
}

\author{
A. Kalliauer \\ Osterreichische \\ Elektrizitätswirtschafts- \\ Aktiengeselischaft \\ 1010 Wien, AUSTRIA
}

\section{ABSTRACT}

The Paper shows the structure of a planning concept for electricity supply systems which, although developed for the Austrian system, is applicable on a general basis to other, more extensive, environmental systems.

\section{INTRODUCTION}

A system to be studied can be characterised by its elements and their relationships. The operation of such a system consists in using its elements to meet a (public) demand. A growth in demand entails an extension of the system and this in turn involves aims that conflict with those of other systems (other systems serving to meet the demand or other environmental systems). This requires consideration of global variables and constraints, which leads to the consideration of more extensive systems.

\section{THE PLANNING PROBLEM}

The elements of the system under study (e.g. power stations) are successively added to the system so as to keep pace with the growing requirements of the system (meeting the demand). The planning problem is the timing of the system extensions. Due to the mutual influences between several extensions we must consider a relatively long planning period. The aim of planning is to make the system fulfil certain tasks at minimum cost, observing the given constraints.

\section{TREATMENT OF THE PROBLEM}

Let the configuration of the elements of a system be denoted by "state". As the state changes with time, it can be expressed as a function of time:

$$
z(t), t \in\left[t_{0}, t_{e}\right],
$$


where $z(t)$ is a vector whose component $z_{i}(t)$ indicates the number of realisations (e.g. power stations placed in operation) of the $i^{\text {th }}$ element at time $t$; if $z_{i}$ denotes a particular project, either $z_{i}(t)=0$ or $z_{i}(t)=1$; if it is a standard unit (a power station type that can be realised any number of times), then $z_{i}(t)=k$ with $k$ being a nonnegative integer.

e.g. $z(t)$ for some $t$ :

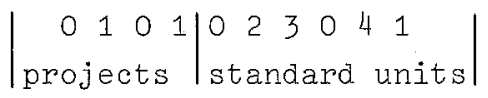

We are trying to find $z^{*}(t)$ with $\mathcal{K}\left(z^{*}\right)=\min _{z \in D}(K(z))$.

Let $\quad \Delta z(t):=\lim _{\varepsilon \rightarrow+0}(z(t)-z(t-\varepsilon))$.

$$
\mathcal{K}(z)=K^{o p}(z)+K^{\text {inv }}(\Delta z)
$$

The dependence of $\mathcal{K}$ on $\Delta z(t)$ is primarily given by the investment costs of unit additions, or more exactly, the total costs which arise from the decision to extend the system.

The dependence of $\mathcal{K}$ on $z(t)$ is expressed by the operating costs. If $z(t)$ cannot meet the demand subject to the constraints this is expressed by $\mathcal{K}(z)=\infty$.

For a finite number of points $t_{i j}: \Delta z\left(t_{i j}\right)>0$.

Hence we can write:

$$
\mathcal{K}^{i n v}(\Delta z)=\sum_{i} \sum_{j} K_{i}^{i n v}\left(\Delta z\left(t_{i j}\right), t_{i j}\right) \text {. }
$$

Let $y_{z}(t)=\left(y_{1}(t), \ldots, y_{i}(t), \ldots, y_{n}(t)\right)$ denote the vector of output of components ("operation variables").

We have to include in our consideration the optimal operating costs:

$$
\mathcal{K}^{\text {op* }}(z)=\mathcal{K}^{\text {op }}\left(z, y_{z}^{*}\right)=\min _{y_{z} \in Y} \mathcal{K}^{\text {op }}\left(z, y_{z}\right) \text {. }
$$

For the purpose of studying the operation of the system we subdivide the study period into intervals at whose length is based on the longest operating cycle in the system (e.g. one year in the Austrian hydro system). With respect to system operation, these time intervals can be regarded as being independent and we can study the relationships within each interval $T_{j}$ separately.

$$
T_{j}=\left[t_{0}+(j-1) * \Delta t, t_{0}+j * \Delta t\right]
$$


then:

$$
K^{o p *}(z)=\sum_{j} K_{T_{i}}^{o p}(z)=\sum_{j}\left(\min _{y_{z} \in Y_{j}} K_{T_{j}}^{o p}\left(z, Y_{z}\right)\right)
$$

with $y_{j}<Y$

As in most of the other methods of treating this problem (see e.g. $(1),(3),(4)$ ), we make this problem a discrete one with respect to these intervals and we apply a discrete Dynamic Programming algorithm. This means that we allow changes in state only at the times $t_{0}, t_{0}+\Delta t$, $t_{0}+2 \times \Delta t_{1} \ldots$ and calculate the operating costs for each interval $\left[t_{0}+(j-1) \cdot \Delta t, t_{0}+j \cdot \Delta t\right]$ separately.

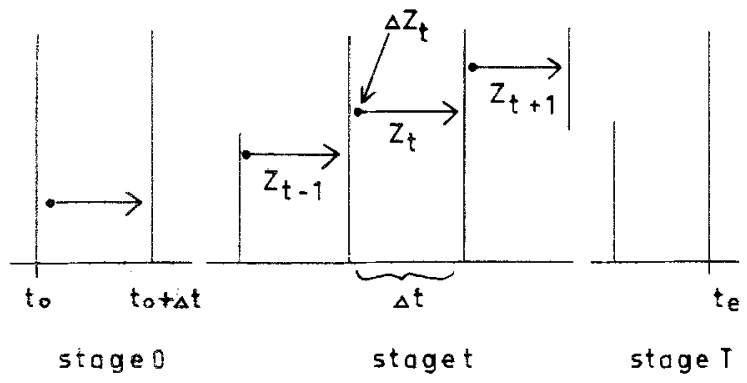

Let $z_{t} \in D_{t}$ and $z_{t}=z_{0}+\sum_{\tau=1}^{t} \Delta z_{\tau}$

The problem can be written:

$$
\min _{\tau=1, \ldots, T}\left(\sum_{\tau=0}^{T} K_{\tau}^{o p^{*}}\left(z_{\tau}\right)+\sum_{\tau=1}^{T} K_{\tau}^{\text {inv }}\left(\Delta z_{\tau}\right)\right)
$$

Now define:

$$
K_{t}\left(z_{t}\right) \min _{\Delta z_{\tau}, \tau=1, \ldots t}\left(\sum_{\tau=0}^{t} K_{\tau}^{o p^{*}}\left(z_{\tau}\right)+\sum_{\tau=1}^{t} K_{\tau}^{i n v}\left(\Delta z_{\tau}\right)\right)
$$

then:

$$
K_{t}\left(z_{t}\right)=\min _{\Delta z_{t}}\left(K_{t}^{\text {inv }}\left(\Delta z_{t}\right)+K_{t-1}\left(z_{t-1}\right)\right)+K_{t}^{o p *}\left(z_{t}\right)
$$

Since hydro power accounts for a large proportion of the Austrian system, the operation model is very complex. To deal both with the questions of long-term planning and with questions of detail, two programs have been developed:

(a) an extension planning program with special routines for defining the states, with a simplified nonoptimising operatio simulation model checked with the help of program (b). 
(b) an operation scheduling program which can solve large-scale Nonlinear Programming problems and is used to solve questions of detail (especially for the hydro system), and to assist in planning by means of model (a).

4. STRUCTURE OF THE EXTENSION PLANNING MODEL (Fig. 1)

Let $z_{t}=\left(z_{1 t}, \ldots z_{i t}, \ldots z_{\text {ht }}\right)$ denote a state at $t$ with $1 \leq t \leq T$.

We now must generate all the $z_{t} \in D_{t}$ :

This is done by using a GENER model, which "sieves out" feasible states, $i . e$. those which satisfy the given constraints associated with stage $t$. other models are now applied to the sets of states so generated, yielding results which we associate with each state. Thus e.g.: The SECUR model determines a security level of a state; or the SIMUL model determines the operating cost over a year calculated on the basis of a suitable but non-optimising operation strategy.

Running the problem with different security levels, we study only the respective associated values and temporarily delete some states if these do not meet the requirements. (We superimpose a mask $\bar{D}_{t} \subset D_{t}$ on the sets of states). In the case of running the problem with different parameters, some of the models need be run only once. In each case, however, the DP algorithm must be run.

This is done in the STEP model:

It serves to associate in each DP step from stage $t-1$ to stage $t$ the total cost value $K_{t}\left(z_{t}\right)$ with each $\boldsymbol{z}_{t}$. These values are the optimal costs in relation to state $z_{t}$ if state $z_{t} \in \bar{D}_{t}$ has been reached by an optimal. path through the stages 1 to $t$. The optimal extension alternative results from the optimal path to $z_{T}^{*}$ of the last stage with $K_{T}\left(z_{T}^{*}\right)=\min _{z_{T} \in \bar{D}_{T}} K_{T}\left(z_{T}\right)$.

5. OPERATION SCHEDULING OPTIMISATION MODEL

As stated earlier, large-scale systems must be studied. The relationships between their elements can usually be subdivided, in a multistage manner, into "global" and "local" ones, that is to say, we can subdivide the total system into subsystems (as e.g. overall generating system and hydro power stations on a certain river), which in turn contain local relationships. These can again be subdivided into global and local ones. Thus we obtain a multi-level structured system model. 
Now consider the operation scheduling model to treat questions of detail.

The dimension of the operation model results from the great number of intervals of which the optimisation period consists, and from the fact that we express the period (year) by some characteristic daily load curves. We can derive the following NLP problem:

The thermal subsystem provides a cost basis for evaluating the hydro system. This results in the following objective function:

$$
\max \sum_{t \in T_{y}} v_{t}\left(P H_{t}\right)
$$

where $v_{t}$ is a noninear evaluation function for the hydro capacity

$\mathrm{PH}_{t}$ and $P H_{t}=\sum_{i \in \mathcal{U}} \mathrm{PH}_{i t}$, where $\mathrm{PH} \mathrm{H}_{i t}$ denotes the capacity of power plant

$i \in U$ at time interval $t$.

Let $Q_{i t}$ be the flow rate at power station $i$ at time interval $t$. Then we can write $P H_{i t}=f_{i}\left(Q_{i t}\right)$.

For $Q_{i t}$, we have to consider lower and upper bounds

$$
Q_{i_{\text {min }}} \leqslant Q_{i t} \leqslant Q_{i_{\text {max }}} \quad \forall i \in U, \forall t \in T_{y} .
$$

Let us define a storage - unit - incidence matrix as shown in Fig. 2. Let $V_{k t}$ be the volume of storage node $k$ at the beginning of the time interval $t$, then $V_{k t}=V_{k 0}+\sum_{i=1}^{t-1} \Delta V_{k \tau}$ with $\Delta V_{k t}=\sum_{i \in U}\left(-i c_{k i}\right) \times Q_{i t}$. The $V_{k t}$ are constrained by $V_{k_{\text {min }}} \leqslant V_{k t} \leqslant V_{k \text { max }}$. This results in a NLP problem in the variables $Q_{\text {it }}$ with the linear constraints resulting from those of the $V_{k t}$.

Let us now consider these constraints: If we are faced with a structured problem, this is expressed by the ic -matrix, which might e.g. take the following form:

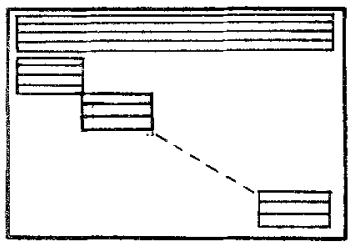


That means that we can subdivide the nodes $k \in K$ into

(1) coupling storage nodes $k \in K_{c}$ and

(2) storage nodes in subsystem $S_{i}: k \in K_{S_{i}}$.

Since in hydro power systems individual storages are scheduled on different cycles (daily, weekly and yearly cycles), we can also define "subsystems" with respect to time, because the constraints for the $V_{k t}$ do not extend beyond the operation period of the power stations.

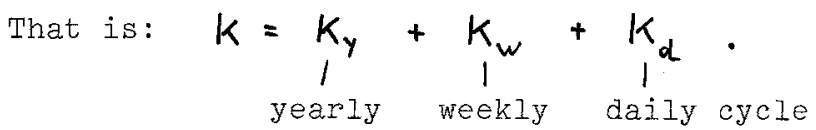

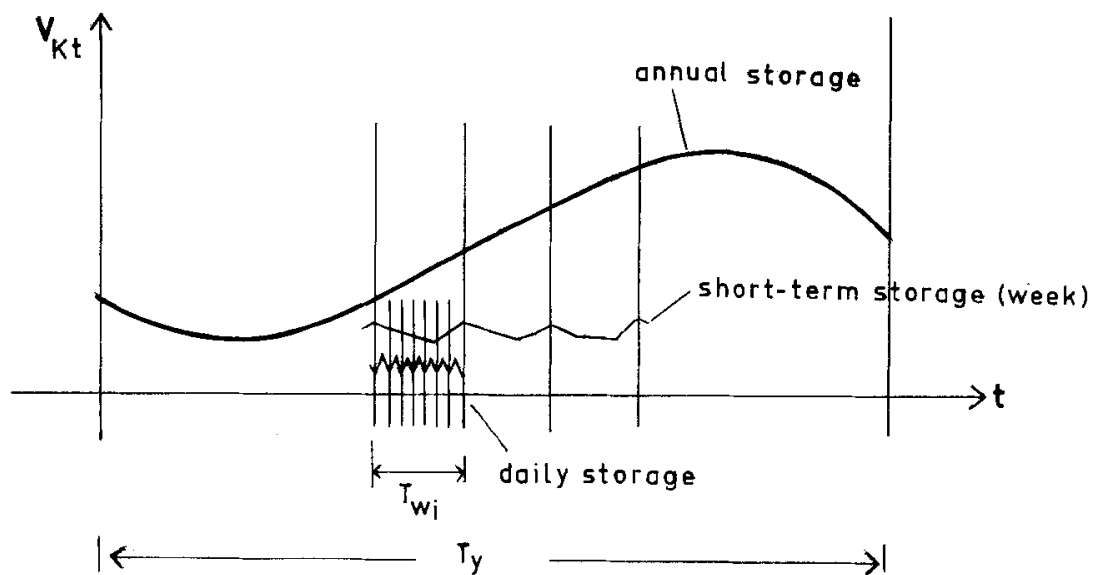

Let: $\quad K_{d c}:=K_{d} \cap K_{c}, K_{d s_{i}}:=K_{d} \wedge K_{s_{i}}$.

Thus we obtain a multi-level structure with coupling constraints as shown in Fig. 3.

\section{APPLICATION OF A MULTI-LEVEL ALGORITHM}

The above NLP problem has been handled by a method taking advantage of the structure of the constraint matrix. The projection $p$ of the gradient $g$ is used as a feasible direction. Instead of calculating the projection vector by the projection matrix $P=I-Q\left(Q^{\top} Q\right)^{-1} Q^{\top}$ and $p=P g$ where $Q=\left(q_{1}, \ldots q_{m}\right)$ is the matrix of active constraints (as e.g. in Rosen's Gradient Projection Method), we use orthogonal vectors $\tilde{q}_{1}, \ldots, \tilde{q}_{m}$, which form a basis for the Iinear space $L\left(q_{1}, \ldots, q_{m}\right)$. 
The projection vector $p$ can be expressed as follows:

$$
p=q-\sum_{i=1}^{m} \tilde{\alpha}_{i} \cdot \tilde{q}_{i}, \quad \tilde{\alpha}_{i}=\left(g, \tilde{q}_{i}\right) /\left|\hat{q}_{i}\right|^{2},
$$

where $(a, b)$ denotes the inner product of vectors $a, b$.

The advantage of the given structure is that constraints from parallel subsystems (i.e. from subsystems whose variable vectors have disjoint index sets) are a priori orthogonal.

\section{REFERENCES:}

(1) Dale, K.M.:

Dynamic Programming Approach to the Selection and Timing of Generation-Plant Additions. Proc. IEE, Vol 113 (1966), No 5.

(2) FeBl,K., A. Kalliauer und G. Schiller:

Die Anwendung von Optimierungsverfahren zur Kraftwerksausbauplanung. ÖZE, 27.Jg. (1974), H. 10.

(3) Neumann, G.:

Optimierung des Ausbaues von Energieversorgungssystemen. VGB Kraftwerktechnik 53, 1973, H. 7 .

(4) Datman, E.N., and I.J. Hamant:

A Dynamic Approach to Generation Expansion Planning. Power Apparatus and Systems, 1973, No 6.

(5) Rosen;J.B.:

The Gradient Projection Method for Nonlinear Programming. Part I. Linear Constraints. SIAM 8, 1960. 


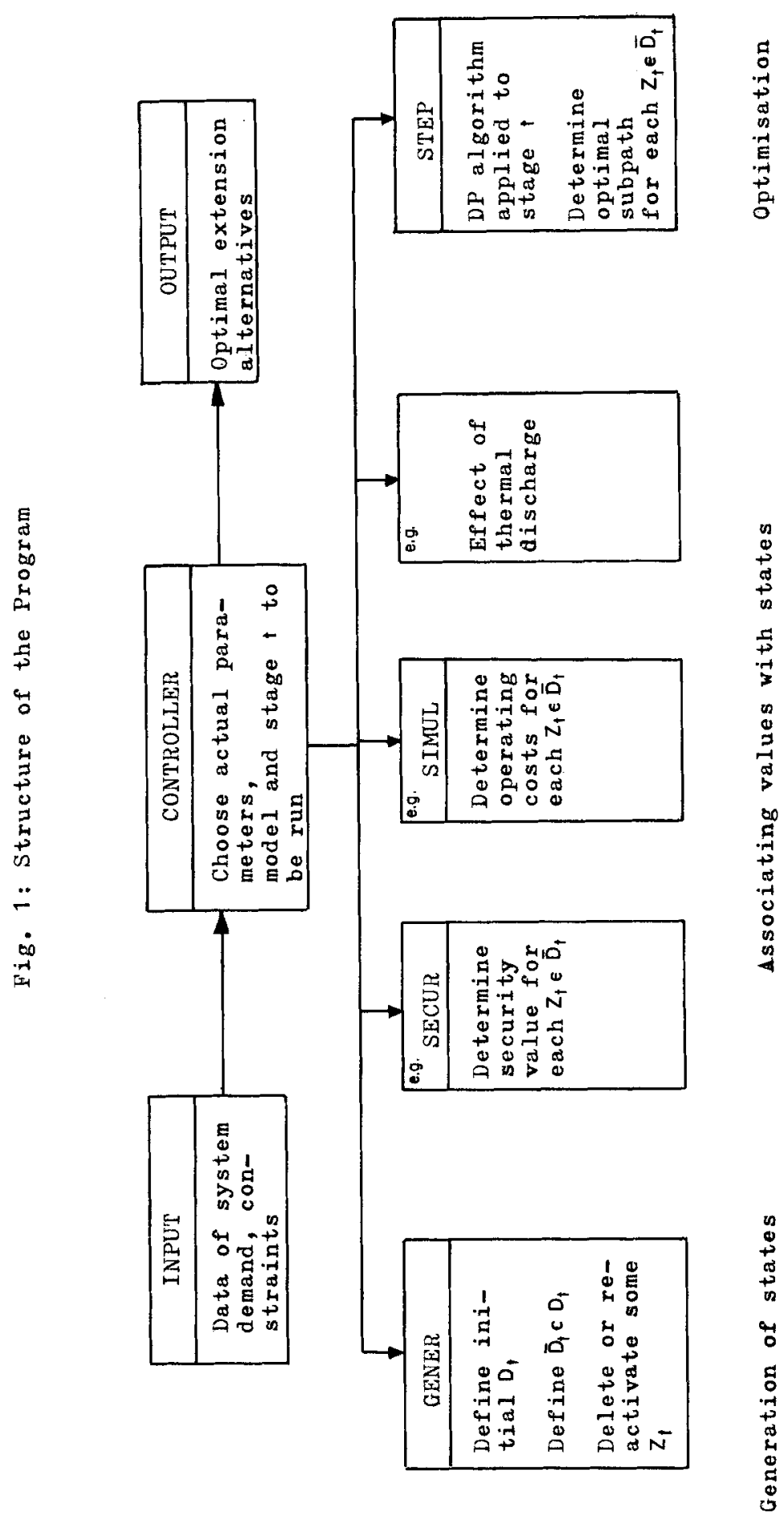



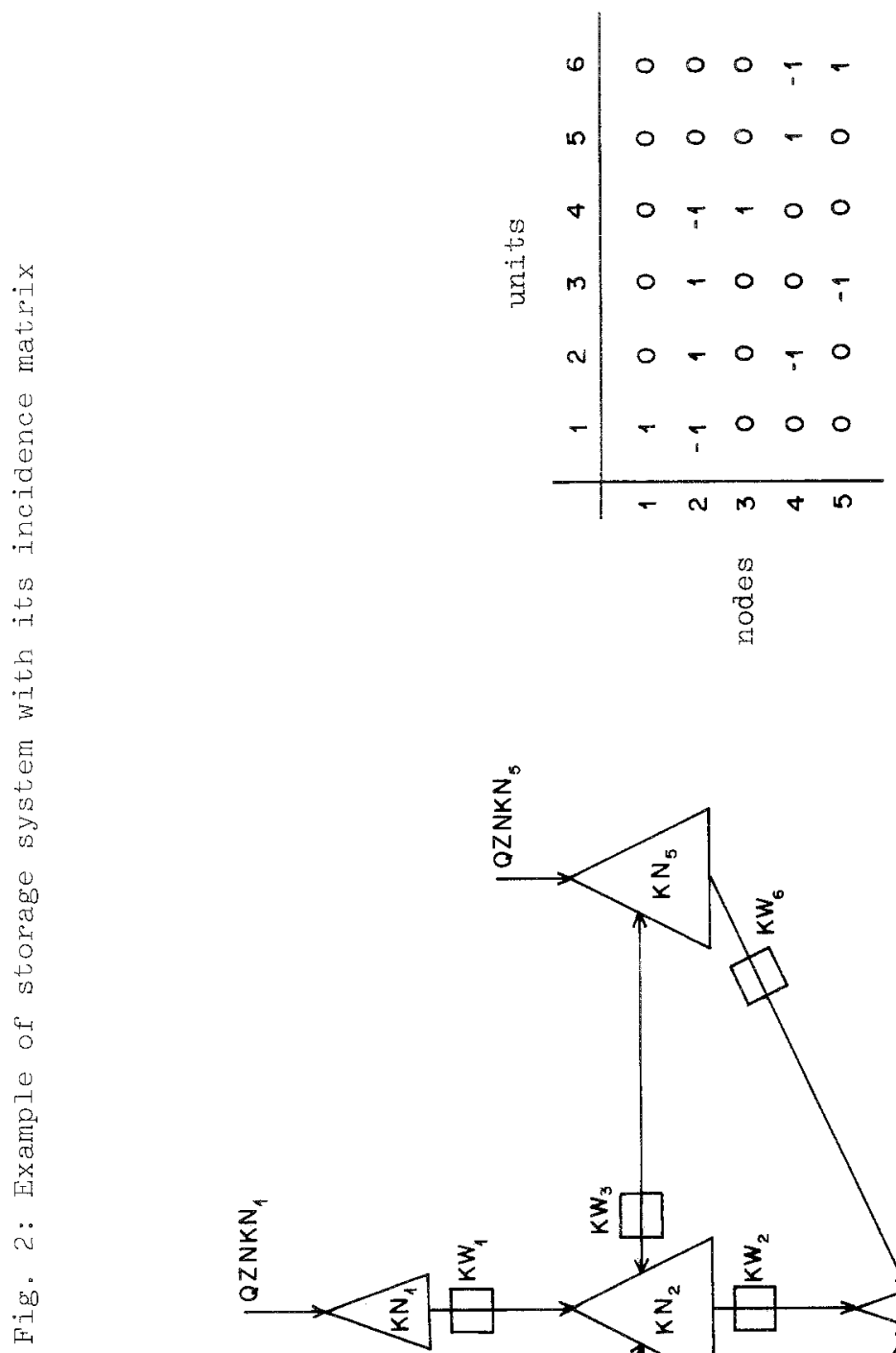

0
0
0
0
0

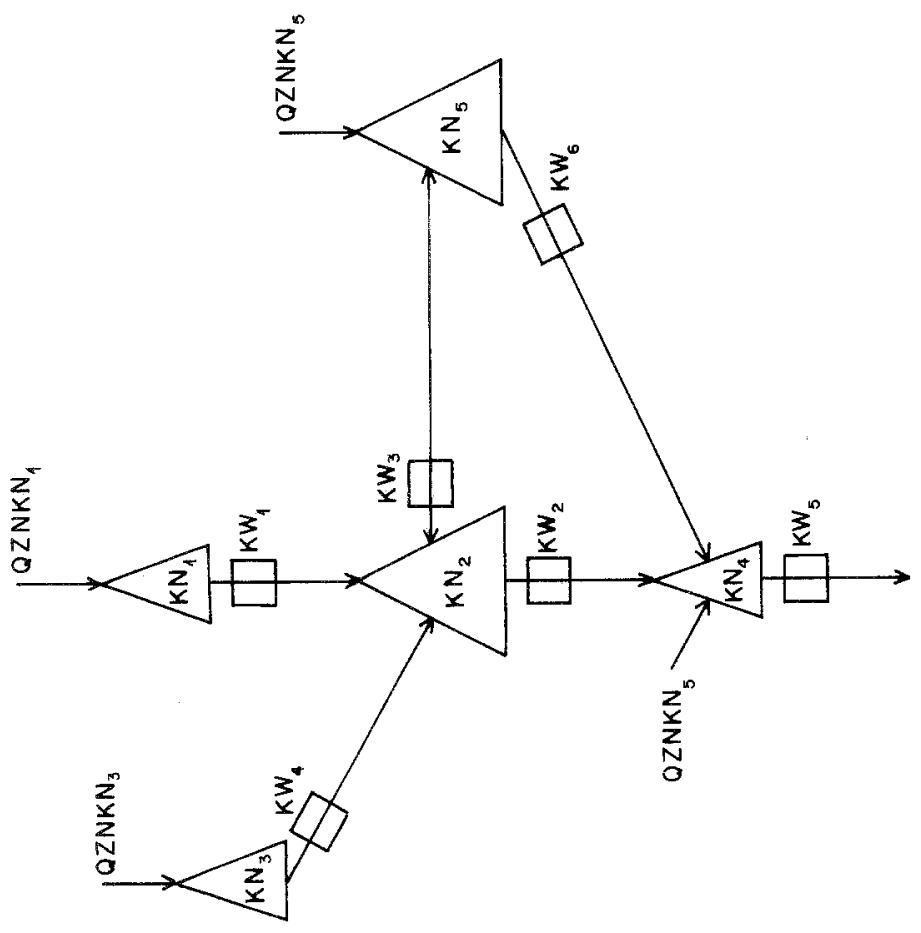




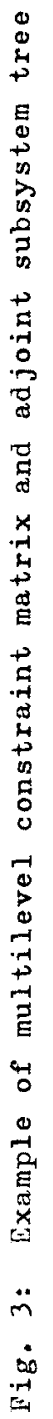
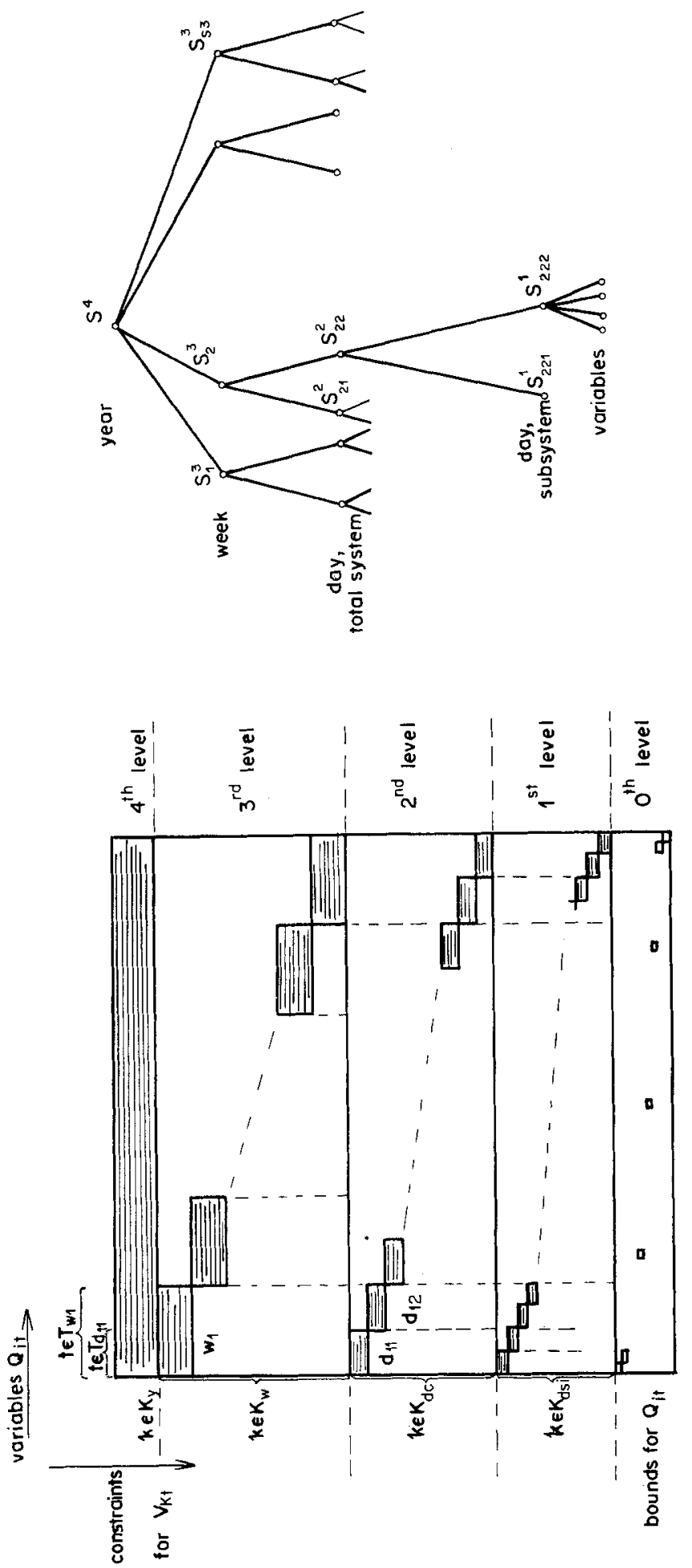